\title{
Heat transfer rate within non-spherical thick grains
}

\author{
Florian Huchet ${ }^{1, *}$, Patrick Richard ${ }^{1}$, Jules Joniot $^{1}$, and Laurédan Le Guen ${ }^{1}$ \\ ${ }^{1}$ LUNAM, Université de Nantes, IFSTTAR, MAST dpt, GPEM, CS4, 44344 Bouguenais, France
}

\begin{abstract}
The prediction of the internal heat conduction into non-spherical thick grains constitutes a significant issue for physical modeling of a large variety of application involving convective exchanges between fluid and grains. In that context, the present paper deals with heat rate measurements of various sizes of particles, the thermal sensors being located at the interface fluid/grain and into the granular materials. Their shape is designed as cuboid in order to control the surface exchanges. In enclosed coneshaped apparatus, a sharp temperature gradient is ensured from a hot source releasing the air stream temperature equal to about $400^{\circ} \mathrm{C}$. Two orientations of grain related to the air stream are considered: diagonally and straight arrangements. The thermal diffusivity of the grains and the Biot numbers are estimated from an analytical solution established for slab. The thermal kinetics evolution is correlated to the sample granular mass and its orientation dependency is demonstrated. Consequently, a generalized scaling law is proposed which is funded from the effective area of the heat transfer at the grain-scale, the dimensionless time being defined from the calculated diffusional coefficients.
\end{abstract}

\section{Introduction}

The response to the temperature variation of the granular assembly surrounded by hot fluid stream is currently encountered in the multiphase flows. Indeed, many applications involving different size and geometry of grains submitted to a convective transfer are emphasized in chemical and materials science or civil engineering domains dealing respectively with various types of gas/solid interaction such as the packed bed [1], the vibrofluidized bed [2] or the rotor-stator system [3].

Discrete element simulations are undoubtedly a relevant tool to study such systems. A common assumption is to consider that grains have a uniform internal temperature, i.e. their Biot number, defined as $B i=h d / 2 k_{s}$ (where $h$ is the convective heat transfer, $k_{s}$ the thermal conductivity and $d$ the grains size) is much lower than 1. Thus, the $B i$ number informs about the use of the lumped capacitance assumption, these latter hypothesis being applicable to the lowest values corresponding to small particle size $(\mathrm{Bi}<<1)$. In that latter case, heat flux is transferred instantaneously from the external layer of the particles to the bulk. The sense of the heat transfer is inverted for hot particles submerged into a fresh fluid. One can refer to the examples of the combustion of char [4] or lime shaft kiln simulation [5] where this assumption can be not relevant. Although it can be justified in many practical cases, this assumption remains invalid in the case of large grains. In that context, the present paper deals with experimental study of the unsteady conduction of non-spherical thick grain submitted to intense temperature gradient in two orientations related to the fluid stream (see fig. 1). The establishment of a simple thermal law devoted to nonspherical particle has a great potential for its implementation in Euler-Lagrange code.
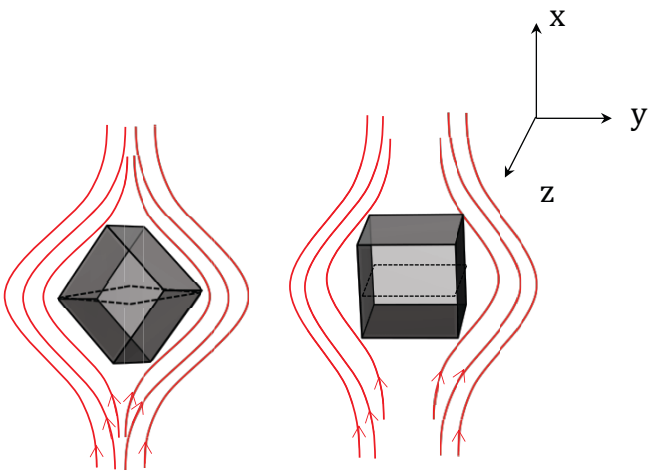

Fig. 1. Two geometrical arrangements perpendicularly to the heat stream are tested, one in diagonal offering an augmented exchange surface while the second is set in the straight configuration offering only one face to the hot stream. The heat transfer area being represented in solid line.

\section{Experimental set-up}

Rock material (gneiss) is chosen as a model of granular materials. Carefully prepared from milling process in order to be designed as cuboid shape, four samples of different size are made (see picture in fig. 2.a. and table 1) to control the surface exchanges. The experimental apparatus is composed of an enclosed cone-shaped system, which controls the air hot stream in upward direction from a lab-scale boiler located below the granular sample. A grid composed of ultra-fin steal wires ( $1 \mathrm{~mm}$ in diameter) is disposed at the interface between

\footnotetext{
* Corresponding author: florian.huchet @ifsttar.fr
} 
the hot source and the measurement area reducing any disturbance between the grain and the hot stream $\left(d_{\text {material }}>>d_{\text {steal wire }}\right)$. The heat flux is controlled from a burner at zero-pressure gradient (i.e. no imposed flow rate), only the buoyancy is responsible of an upward heat flux toward the granular sample. According to $[6,7]$, the stream velocity in the vicinity of the rock is estimated to $4.3 \mathrm{~m} / \mathrm{s}$.

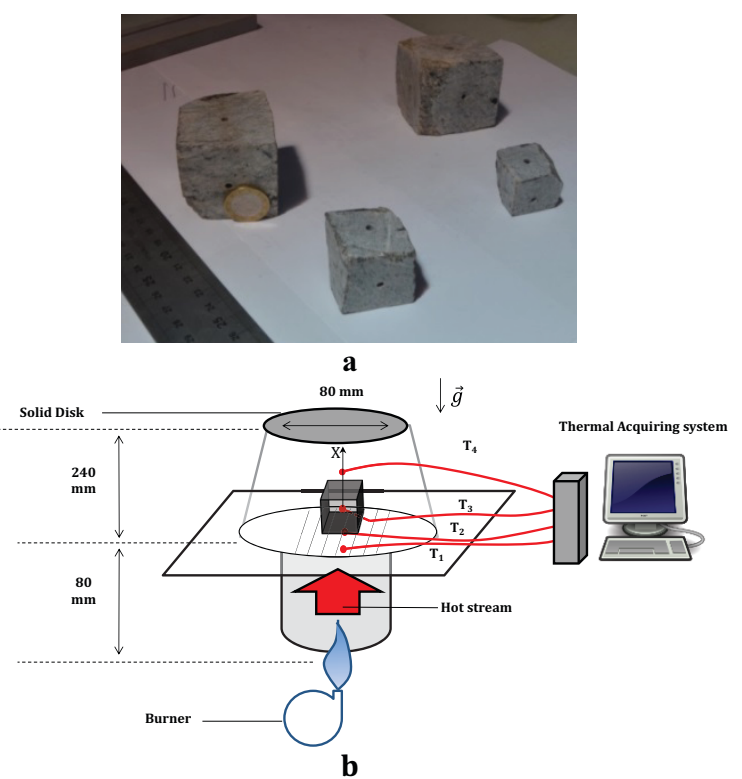

Fig. 2a. Rocks materials with four different sizes are milled while holes are bored at the center of the surface samples. Fig. 2b. The granular sample is composed of two holes. One is accomplished in the near-wall surface to set one K-thermal probe $\left(\mathrm{T}_{2}\right)$ at $1=1 \mathrm{~mm}$. The second is drilled in the heart of the granular medium close to the central location $\left(\mathrm{T}_{3}\right)$. The rest of the thermal probes are located above $\left(\mathrm{T}_{4}\right)$ and below the grain $\left(\mathrm{T}_{1}\right)$. The thermoregulation is ensured from a burner from the thermal probe, $\mathrm{T}_{1}$, ranged between $350^{\circ} \mathrm{C}$ and $450^{\circ} \mathrm{C}$.

The surface of the granular matter « seen » by the hot fluid is determinant to characterize the interaction between fluid and grain, especially for non-spherical particles. The state of art related to that topic remains confused between the works established in the case of the forced convection $[8,9]$ or the free convection $[10$, 11]. In that context, two orientations were investigated, one located diagonally with respect to the fluid stream while one is straight with respect to the fluid stream. An effective surface, $S_{\text {eff, }}$, is required to give an insight of the shape factor. Defined as the projected area for the diagonal orientation, one face as the inner heat transfer area is considered for the straight orientation case (see sketch in figure 2.b.). Note that the aspect ratio between the two orientations is $\sqrt{2}$. The table 1 recaps for each sample the ratio between the effective surface and the overall area of transfer, $S$, the mass of the grain, and the characteristics length of the thermal diffusivity between the inner face and the center of the sample. Afterward a particular attention of the positioning of the probes, the cone-shape is systematically contained in closed-volume, from a solid disk, before the beginning of the experiments. Then, all the experiments were conducted in confined media with a temperature regulation of the hot stream between $350^{\circ} \mathrm{C}$ and $450^{\circ} \mathrm{C}$ controlled from the first probes, $\mathrm{T}_{1}$.

Table 1. Physical characteristics of each cuboid granular sample.

\begin{tabular}{|l|c|c|c|c|}
\hline \multicolumn{1}{|c|}{$\begin{array}{c}\text { Granular } \\
\text { sample }\end{array}$} & $\mathbf{S}_{\mathbf{1}}$ & $\mathbf{S}_{\mathbf{2}}$ & $\mathbf{S}_{\mathbf{3}}$ & $\mathbf{S}_{\mathbf{4}}$ \\
\hline Mass (g) & 287 & 288 & 91.5 & 50 \\
\hline $\begin{array}{l}\text { Area of } \\
\text { transfer, S } \\
\left(\mathrm{cm}^{2}\right)\end{array}$ & 127 & 138.5 & 58 & 39.5 \\
\hline $\begin{array}{l}\text { Diffusional } \\
\text { tickness, L (cm) }\end{array}$ & 2.30 & 2.40 & 1.55 & 1.28 \\
\hline $\begin{array}{l}S_{\text {eff }} / S \\
\text { Diagonal } \\
\text { orientation }\end{array}$ & 0.264 & 0.235 & 0.262 & 0.277 \\
\hline $\begin{array}{l}S_{\text {eff }} / S \\
\text { Straight } \\
\text { orientation, }\end{array}$ & 0.187 & 0.166 & 0.185 & 0.196 \\
\hline
\end{tabular}

For each experiment, four thermocouples are positioned (see fig. 2.b) such as: (i) Below the grain to measure the hot stream, $\mathrm{T}_{1}$, (ii) Through a thin hole, $1=1$ $\mathrm{mm}$ in depth at the inner face to measure the wall temperature, $\mathrm{T}_{2}$ (iii) Into a deeper hole until the center of the sample to measure the bulk temperature, $\mathrm{T}_{3}$, corresponding to the diffusion length, $\mathrm{L}$, (iv) Above the sample to measure the gas temperature, $\mathrm{T}_{4}$. Note that the volume devoted to the thermocouples is small (less than $2 \%$ of the total volume). Consequently we assume that its effect on the conductivity is negligible.

As aforementioned, the thermal apparatus is composed of four thermocouples of type $\mathrm{K}$ connected to an acquiring system. The sampling frequency is equal to 1 hertz and the experiment is performed during 10 minutes. An example of the temperature time-traces is presented in the figure 3 for each probe location. A sharp temperature increase is noticeable for $\mathrm{T}_{1}$ probe. This shows the beginning of the experiment. One can see the heat transfer competition between convection and radiation modes. Indeed, $\mathrm{T}_{1}$ and $\mathrm{T}_{2}$ probes locations are close to the hot stream compared to $\mathrm{T}_{4}$ probe location into the fluid flow.

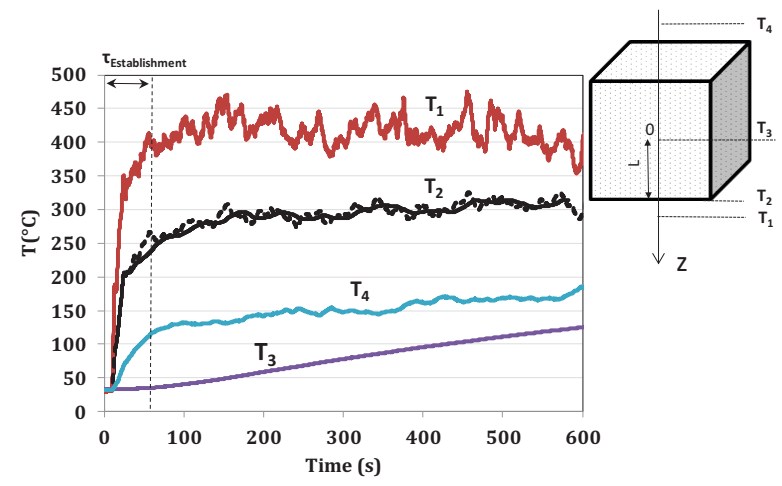

Fig. 3. Experimental apparatus controlled by a burner whose the first thermal probe $T_{1}$ is adjusted around a value ranged between 350 and $400{ }^{\circ} \mathrm{C}\left\{\mathrm{T}_{1}>\mathrm{T}_{2}>\mathrm{T}_{4}>\mathrm{T}_{3}\right\}$. The signals measured from probes close to the heat flux are subject of noise imputed to the intense thermal gradient. A slidingaverage window is able to filter the fluctuations (see continuous black line). 
The time of thermal establishment, $\tau_{E}=60 \mathrm{~s}$ is necessary to reach a constant temperature $\mathrm{T}_{1}$ close to 400 ${ }^{\circ} \mathrm{C}$. $\mathrm{T}_{3}$ corresponds to the bulk temperature of the granular material; its variation is slow and undisturbed since the kinetic is governed by the thermal diffusion. The elevated temperature fluctuations are filtered in order to obtain a suitable signal to process of the data analysis. Then, according to the temperature variation, different sliding-average windows are applied in order to filter the fluctuations of $\mathrm{T}_{2}(\mathrm{t})$ as seen in fig. 3 .

\section{Results and discussion}

\subsection{Heat Transfer properties}

The unsteady heat conduction equation in anisotropic medium can be expressed in dimensionless form as:

$$
\frac{\partial T}{\partial t}=a\left(\frac{\partial^{2} T}{\partial r^{2}}+\frac{n}{r} \frac{\partial T}{\partial r}\right)
$$

With $\mathrm{n}=0,1$ and 2 for slab, infinite cylinder and sphere respectively, and $a$ the thermal diffusivity.

The solution for a slab (with $r=X$ ) is solved and given in function of the Fourier number $\left(F o=t / a L^{2}\right)$ such as:

$$
\begin{gathered}
T(X, t)=\frac{T_{1}-T_{X}}{T_{1}-T_{X, t=0}}= \\
\sum_{i=1}^{n} \frac{2 \sin \beta_{n} \cos \left(\beta_{n} X / L\right)}{\left(\beta_{n}+\sin \beta_{n} \cos \beta_{n}\right)} \exp \left(-B_{n}{ }^{2} F o\right)
\end{gathered}
$$

Where $B_{n}$ is the $\mathrm{n}^{\text {th }}$ positive root of:

$$
\beta \tan \beta=B i
$$

Consequently, the thermal diffusivity, $a$, can be assessed by minimizing the relative error between the analytical model calculated from the equation (11) and the temperature measurements (with $T_{X=L}=$ $T_{3}$ ) performed for each sample at a given orientation. The average thermal diffusivity of our materials is found equal to $2.90 \times 10^{-7} \mathrm{~m}^{2} / \mathrm{s}$ with a relative error equal to 6.5 $\%$ calculated at $\mathrm{Fo}>0.2$.

Thus, the ratio method has been applied to assess the Biot number of each sample. As reported by [12], it is based on the evaluation of $T(X, t)$ at any location in such way that the equation (11) can be reduced at the first order for the Fourier number superior to 0.2 :

$\forall X: \frac{T_{1}-T_{X}}{T_{1}-T_{X, t=0}}=\frac{2 \sin \beta_{n} \cos \left(\beta_{n} X / L\right)}{\left(\beta_{n}+\sin \beta_{n} \cos \beta_{n}\right)} \exp \left(-\beta_{n}{ }^{2} F o\right)$

By taking into account two positions of measurement into the grains, it comes:

$$
\left\{\begin{array}{c}
\frac{T_{1}-T_{X=0}}{T_{1}-T_{X, t=0}}=\frac{2 \sin \beta_{n}}{\left(\beta_{n}+\sin \beta_{n} \cos \beta_{n}\right)} \exp \left(-\beta_{n}{ }^{2} F o\right) \\
\frac{T_{1}-T_{X=L-l}}{T_{1}-T_{X, t=0}}=\frac{2 \sin \beta_{n} \cos \left(\beta_{n}(L-l) / L\right)}{\left(\beta_{n}+\sin \beta_{n} \cos \beta_{n}\right)} \exp \left(-\beta_{n}{ }^{2} F o\right)
\end{array}\right.
$$

With $\mathrm{T}_{1}$, the inlet temperature condition, $T_{X=0}=T_{3}$ and $T_{X=L-l}=T_{2}$

The ratio between (14) and (15) defines:

$$
Z=\frac{T_{1}-T_{2}}{T_{1}-T_{3}}=\cos (\beta(L-l) / L)
$$

From a geometrical identification showed in figure 4.a, $\beta$ is obtained from the equation (16) and the Biot number is approximated from the equation (12). As expected, $B i$ is found superior to 1 with the major values imputed to the straight orientation. This latter result corresponds slightly to a heat transfer augmentation related to its geometric configuration with respect to the heat flux.

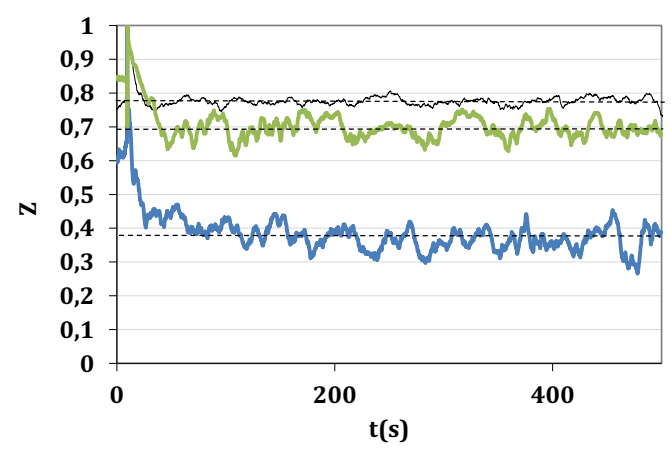

a

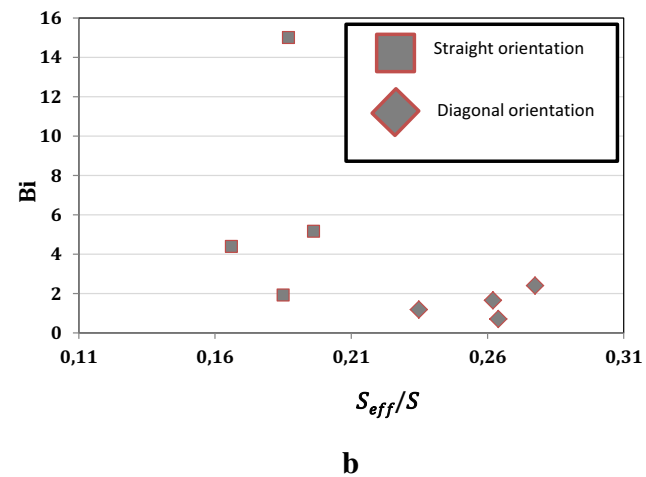

Fig. 4 a. Left: Examples of experimental $Z$ values. b. Biot number assessed for each sample.

\subsection{Scaling based on the dimensionless surface}

To damp the anisotropy effect on the Biot numbers results, a dimensionless criterion is necessary. Thus, the product of the dimensionless temperature $T^{+}$and the shape factor, $S^{+}$is, here, proposed such as:

$$
S^{+} T^{+}=\frac{S_{\text {eff }}}{S} \frac{\left|T_{\text {bulk }}-T_{\text {wall }}\right|}{T_{\text {wall }}}=\frac{S_{\text {eff }}}{S} \frac{\left|T_{3}-T_{2}\right|}{T_{2}}
$$

Such dimensionless parameters plotted versus time give still large discrepancies (not shown here). Besides, the time must be converted into a dimensionless number. Inspired to the Fourier number, this is defined from the diffusivity coefficient $\left\{a=\rho c_{p} / \lambda\right.$ en $\left.\mathrm{m}^{2} / \mathrm{s}\right\}$ and the characteristics length, L, corresponding to thermal thickness of diffusion (see table 1):

$$
t^{+}=\frac{t}{L^{2} / a}
$$

Consequently, the overall curves $T^{+}=f\left(t^{+}=F o\right)$ are well-scaled from the Fourier Number as seen in the figure 5.a. Two relationships are proposed to correlate the experimental data respectively at the lowest and the highest Fo numbers:

- At Fo $<0.02$ :

$$
\mathrm{T}^{+} \mathrm{S}^{+}=\frac{1}{\alpha} \mathrm{e}^{\left[\ln (\alpha \cdot \text { Fo }) \frac{\beta}{\alpha^{2}}\right]^{\beta}}
$$

with $\beta=5, \quad \alpha=10$ for $0.01<$ Fo $<0.02, \quad$ and $4 \leq \alpha<10$ for Fo $<0.01$;

- At Fo> 0.02 :

$$
\mathrm{T}^{+} \mathrm{S}^{+}=\gamma \mathrm{e}^{-\delta \mathrm{Fo}}
$$


with $0.14<\gamma<0.15,1<\delta<1.5$.

Note that at the lowest Fourier numbers inferior to 0.01 , the physical scaling is not representative of the overall experimental data. In this range of the $F_{O}$ number, the transverse diffusional is more sensitive and a 2-D heat conduction model seems required. Above a $F o$ number equal to 0.015 , it seems quite reasonable to keep a 1-D model as the equation (10).

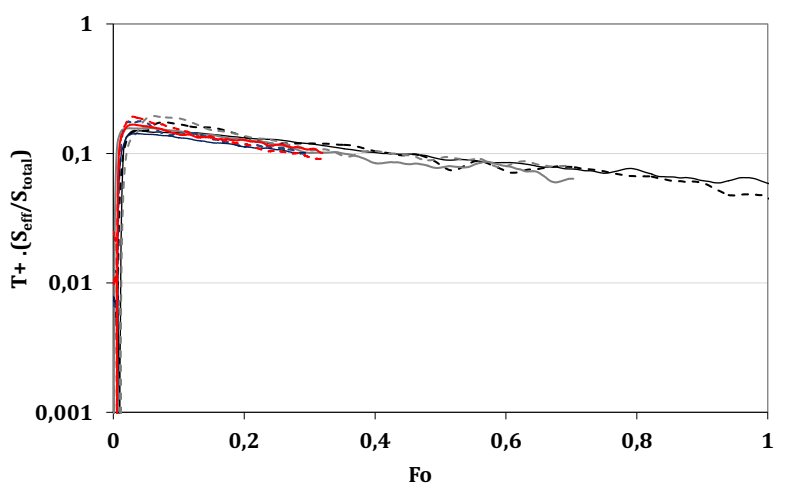

a

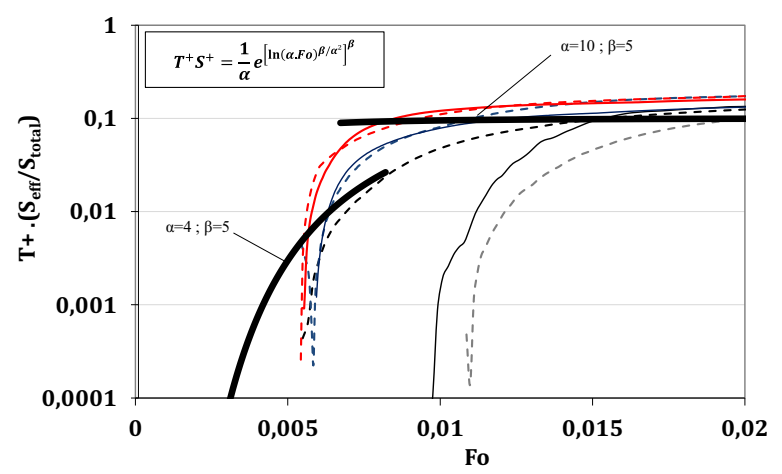

b

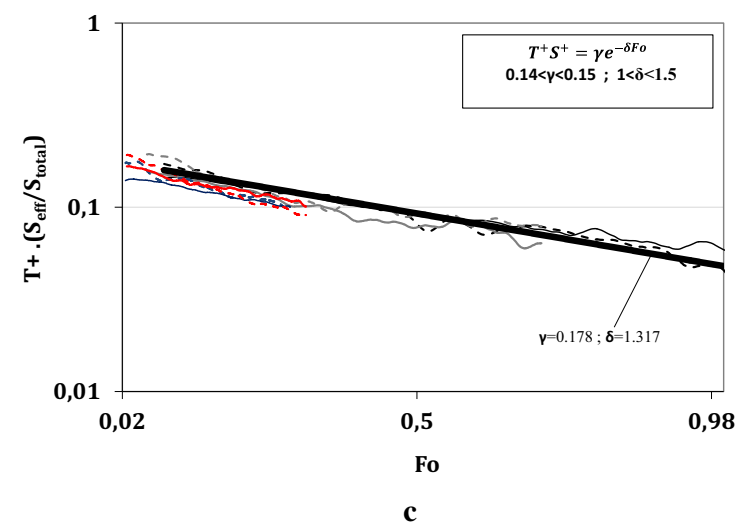

Fig.5.a. Dimensionless representation of the conduction phenomena into non-spherical grain with various orientations. b. Focus at the low Fourier number c. Focus at the high Fourier number. heat flux. Two fluid flow orientations have been explored such as the grains, from the same origin, are oriented diagonally or straight with respect to the heat flux. The experimental results show that the $B i$ number is, as expected, superior to 1 . Ranged between 0.7 and 15, the highest values of $B i$ are connected to the straight orientation. To damp the geometrical configuration upon the heat transfer coefficients, our results are scaled from new original geometrical parameters based on the effective area of transfer as shape factor, which is found representative of the overall measured materials temperature in terms of $T^{+} S^{+}=f(F o)$. Two correlations are expressed in the range of the $F o$ numbers such as:

- At $F_{O}<0.02$ :

$$
\begin{gathered}
\left.\mathrm{T}^{+} \mathrm{S}^{+}=\frac{1}{\alpha} \mathrm{e}^{\left[\ln (\alpha \cdot \mathrm{Fo}) \alpha^{\frac{\beta}{2}}\right.}\right]^{\beta} \\
\text { At } F o>0.02: \\
\mathrm{T}^{+} \mathrm{S}^{+}=\gamma \mathrm{e}^{-\delta \mathrm{Fo}}
\end{gathered}
$$

Note that at the lowest $F o$ number, the fit is much less representative of the experimental data. This difference is imputed to the transversal diffusion, thus the problem cannot be reduced to one-dimensional model.

\section{References}

1. Yu Y.S., Liu W.Q., An H., Yang F.S., Wang G.X., Feng B., Zhang Z.X., Rudolph V., Int. J. Greenhouse Gas C., 10, 510-519 (2012)

2. Raison C.E., Thomas B., Squires A.M., Powder Tech., 169, 136-142 (2006)

3. Le Guen L., Huchet F., Dumoulin J., Baudru Y., Tamagny P., Appl. Therm. Sci., 54, 131-139 (2013)

4. Zhou H., Flamant G., Gauthier D., Chem. Eng. Sci., 59, 4205-4215 (2004).

5. Bluhm-Drenhaus T., Simsek E., Wirtz S., Scherer V., Chem. Eng. Sci., 65, 2821-2834 (2010)

6. Yan Z., PhD thesis, Université de Poitiers, France (2014)

7. Rouse H., Yih C S., Humphreys H W., "Gravitational convection from boundary source." Tellus 4, 201-210 (1952).

8. Duan Z., He B., Duan Y., Scientific report 5, 12304 doi: 10.1038/srep12304 (2015)

9. Richter A., Nikrityuk P. A., Int. J. Heat Mass Transfer 55, 1343-1354 (2012).

10. Hassani A.V., Hollands K.G.T., J. Heat Transfer 111, 363-371 (1989)

11. Yovanovich, M.M., ASME HTD 82, 121-129 (1987).

12. Awuah G.B., Ramaswamy H.S., Simpson B.K., Food Res. Int., 28 3, 261-271 (1995)

\section{Conclusions}

This work is devoted to the unsteady conduction of cuboid rock materials submitted to an imposed upward 